\title{
Smart Transportation Application using Global Positioning System
}

\author{
Nouf Mohammad Al Shammary and Abdul Khader Jilani Saudagar \\ Information Systems Department, College of Computer and Information Sciences \\ Al Imam Mohammad Ibn Saud Islamic University (IMSIU) \\ Riyadh, Saudi Arabia
}

\begin{abstract}
Significant increase is noticed in the utilization of mobile applications for different purposes in the past decade. These applications can improve any individual's way of life in many aspects such as communication, collaborative work, learning, location services, data collection, exploring, testing and analysis. One of the most interesting mobile applications is using it for tracking by having personal locators. These locators can track children, people on work, the elderly for personal protection etc. The intention behind developing this mobile application is to provide a smart transportation system to it users and to track their movements.

Some of the essential features of this application are

- Getting familiar with the shortest path from source to destination in advance.

- Aware of approximate time of arrival to destination.

- Knowing the capacity of vehicle used for transportation.

- Short Message Service.
\end{abstract}

Keywords-communication; global positioning system; smart application; tracking; transportation

\section{INTRODUCTION}

Managing transportation is a collaborative activity that is based upon sharing and exchanging information. Transportation system needs an easy and effective way to manage their staff and the registered users who are using the transportation services. Staff needs an effective way to know the shortest path to get to the locations, handle the requests of the users etc. Registered users also need to keep up with the staff movements without waiting too early or causing too much delay. The registered users can inform the staff about their absence using short message service. Providing the above mentioned services collectively and managing them from any location is a challenging problem. Mobile technology can be a solution for the above problem using location based applications which serves managing transportation by navigating maps. In recent years, mobile technology revolution and its applications reached to its climax. This revolution resulted in an aggressive competition between the application developers as the developed mobile applications are similar in concepts and are identical in design but are set apart by unique features, offered services to complement the main idea.

Mobile phones are becoming smaller with modern Graphical User Interfaces (GUIs), and are very powerful. In addition, they are now can be used everywhere and at any time (ubiquitous). The advancements and enhancements the hardware went through have made mobiles smaller in size and more effective and efficient. Furthermore, it allows us to include many types of peripherals that are not limited with a specific number [1]. Nowadays, there are three different methodologies to allow smart mobile phones to be capable of utilizing the location and positioning services: 1) The utilization of Satellite Positioning, 2) The utilization of Wi-Fi Positioning and 3) The utilization of Cellular Positioning. When looking at these positioning methodologies in a general way, it is noticeable that they have different characteristics which can be measured by Accuracy, Precision, Power consumption, Latency and Availability as proposed by the researcher Hightower et. al [2].

Tracking service providers ${ }^{1}{ }^{2}$ [3] save the information related to locations in their own database and users are capable of accessing this information. It can be accessed and efficiently utilized upon paying over international telecommunication infrastructure. Furthermore, taking advantage of the SMS availability in mobile networks facilitates users to communicate, transfer information from one place to another. In these types of systems, the users will be more limited since this service has limited flexibility with higher cost.

In [4] the work entitled "GIS Based Public Bus Transport Management System for Nairobi City”, Otieno and Ngigi introduced a public bus transport management system that utilizes Geographic Information Systems (GIS) and Global Positioning System (GPS) technologies and the system completely depends on these technologies. The system is capable of storing the day-by-day information that a public transport vehicles produce in an intension to help in having an efficient and effective management of public transport. This work also demonstrated the way of how the collected tracking data can be utilized to manage the movements of public transport vehicles when it is added and mixed with other types of data from other sources, for instance, vehicle monitoring inventory.

In [5] the researchers had the aim to achieve the following two objectives:

\footnotetext{
${ }^{1}$ http://thenextweb.com/location/2010/08/24/geo-positioning-on-the-iphone4-is-doing-just-fine-without-skyhook-and-google

${ }^{2}$ http://www.engadget.com/2010/09/17/ skyhook-google-forced-motorola-todrop-our-location-service-de/
} 
- Developing an automatic vehicle location system. In addition, GPS, GSM technologies will be necessary for the purpose of information transmission.

- Sending some of information that is related to the locations of vehicles (the focus was on buses) to children's parents through the utilization of SMS.

Global Navigation Satellite System (GNSS) is the primary system in their project that they are building; it utilizes a satellite that is used for positioning and tracking. GNSS is primarily utilized for monitoring numerous kinds of vehicles, for instance, airplanes, cars (including buses) and other kinds of vehicles. In this paper, they considered buses are the most important and common ways for transportation that allow us to move from one place to another. As a result of that, this is the vehicle that this proposed application will deal with mostly. When passengers coming from different places move or travel from one location to another, there is a chance that they may face different types of problems, for instance, knowing the arriving time of a bus so they can use their time wisely as well as knowing the time when to reach the desired bus stop etc. As described by the researchers who proposed this mobile application, the primary goal of this application is to minimize the time that the parents should wait when they are at the bus stop. This can be achieved through sending some information related to the different locations that the buses pass by during their movements to those parents through the utilization of SMS. The idea of this application is providing a mobile application that will benefit passengers to get to a bus stop without wasting any time. In practice the buses are often late due to several reasons, for instance, intense traffic. It would be a very useful idea to be able to build a web application for observing buses movements in real time. The process of observing buses is performed through utilizing manual methods to get the position of a bus, for instance, signals are manually operated by staff that perform the instructions using telephone from the closest bus stop. So, information related to buses movements that is propagated to the public is dependent on manual process. The proposed system only gives the position of a bus but cannot give the exact position of the bus or where it is in a particular moment. To solve this problem, a GNSS based web application is utilized which provides with the exact location of a bus that will be shown on Google Maps as well as the bus speed. Combining GNSS with a web tools such as Google Maps and web browser offers a cheap and useful bus monitoring system that solves several problems.

Other mobile application that is used to find a bus arrival time is called Ride Systems GPS ${ }^{3}$. This system provides passengers with the next bus time, in other words, the time when their next bus will arrive. The most important feature that this mobile application provides is finding stops. Furthermore, it provides the arrival times for a particular route. This application can be used by Android and iPhone operating systems.

In the report [6] entitled "Wireless Global Positioning System Fleet Tracking System at the University at Albany"

\footnotetext{
${ }^{3}$ http://www.pc.pitt.edu/transportation/routes.php
}

prepared by New York State Energy Research and Development Authority, an overview of the project was proposed at the University at Albany that is introduced to make interesting ways to interact with transportation facilities which produces a more attractive option to deal with these facilities. The system includes implementing a GPS Tracking System on the University bus station. This system sends the different locations that the bus passes by to the users of the system. This propagation will be through broadcasting this information to passengers via the internet and a smart phone application. In 2009, the university made a survey related to transportation of the students and staff of the university. From the survey analysis, the results were as follows:

- $73 \%$ of the staff working there and $39 \%$ of the students studying there have used primarily a private vehicle for transportation to and from the university.

- People who have actually utilized the University of Albany bus daily were just $18 \%$ of the students and $2 \%$ of the staff.

Participants to the proposed survey have mentioned that the biggest obstacle behind using the university buses was related to the "convenience" factor. After utilizing the GPS real-time tracking system and installing it, it has been noticed that there was a small increase in the percentage of passengers that utilized the transportation of University of Albany buses.

Mobile tracking applications are very popular nowadays. One of the most well-known location tracking systems is the save and sound system [7]. This system enables parents to monitor their children's locations. The child's phone continuously streams location information to the parent's phone. A secure zone will be created beyond which a child may not travel. If the child leaves this zone, both child and parent receive an audible alert, and the parent can communicate with the child by voice over the phone.

However, the Federal Communications Commission (FCC) of the United States has mandated all wireless service providers to provide an E-911 service whereby the mobile user location is reported when they dial an emergency call (911). This development has helped many location based services (LBS) [8]. Various application systems have emerged for mobile location systems such as location-based mobile tourist services, location-based game and location-based speaker segmentation [9].

Marco Anisetti and colleagues [10] agreed that GPS is not the key element for location-based services because of some issues where GPS is unfeasible in dense urban areas or inside buildings where satellites are not visible from the mobile terminals; they propose the position and motion tracking system (PMTS). PMTS differs from GPS in three respects: i) it exploits only information available to the network itself, ii) it does not require additional hardware and iii) it provides mobility prediction at both the network and the service level.

Some of the previous studies for mobile location systems focus on privacy concerns in using location-based services. Louise and Anind [11] found that, on a scale of 1 to 5, the participants in their study averaged 2.75 for privacy concerns. This study showed that people are not overly concerned about 
their privacy when using location-based services. On the other hand, another study focuses on privacy concerns for location tracking services, which showed that 3 out of 16 participants were highly concerned and the rest were not concerned or didn't mind either way. The final conclusion of this study is that people are more concerned when others can track their location than when their phone reacts to its own location.

Information that can be taken from the GPS is highly needed in many applications. Tracking Pro is one of these applications. The GPS Phone Tracking Pro application makes it simple to track the movements of other mobile devices by allowing observers to find the exact location of the observed users and getting real-time updates about their movements. It is used most of the time to locate any phone that has been misplaced or stolen ${ }^{4}$. Another application is U Safe Tracker application $^{5}$; its ultimate purpose is the safety of the observed users by reporting their locations to the observers. This application uses the mechanism: Set the application to send the observed user's location every 30 minutes or at a specific time only (e.g. 9:00 PM every day).If no location signal is received after 30 minutes (or at a time specified by the observer), the phone's owner may be in danger.

Mobile Tracker $^{6}$ is also an application that keeps track of other mobile phones. Its ultimate goal is tracking lost mobile phones, and it works as follows:

- Create an account so observers can login from anywhere.

- Observers should pick four contacts by providing their mobile numbers.

- When this mobile gets lost or stolen and when the new SIM card is inserted in the mobile, this application automatically sends SMS from the SIM card that is inserted.

- Once the SMS is received, observers can make a complaint on that number that they received SMS from and the mobile will be tracked.

Mobile Number Tracker $^{7}$ is an application that provides information about calls that are received from unknown numbers and observers will be able to find out the location of the caller. The proposed developed application is enhanced version of the existing applications with more features that coordinate the work of three end users (transportation administrator, bus driver, parents and passenger (student)), they use similar techniques and tools but are differ in purpose.

This problem entails the need for mobile application that is able to better utilize the Global Positioning System (GPS) capabilities of a mobile phone and provide services that overcome the currently existing problems. This mobile

\footnotetext{
${ }^{4}$ https://play.google.com/store/apps/details?id=com.fsp.android.c\&hl=en

https://play.google.com/store/apps/details?id=homesoft.app.falcontracker\&hl $=$ en

${ }^{6}$ https://play.google.com/store/apps/details?id=com.nav.mobile.tracker
}

https://play.google.com/store/apps/details?id=com.ViQ.Productivity.MobileN umberTracker\&hl=en application is developed for Imam University Transportation System and tested by transportation administrators, bus drivers, and students of Imam University, Riyadh, Saudi Arabia. It is compared with the existing applications and a comparison table in terms of tools and technologies is as shown in Table 1.

TABLE I. COMPARISON BETWEEN TECHNOLOGIES

\begin{tabular}{|c|c|c|c|c|c|}
\hline & \multicolumn{5}{|c|}{ Systems } \\
\hline & [4] & [5] & 3 & [6] & $\begin{array}{c}\text { Proposed } \\
\text { System }\end{array}$ \\
\hline GPS & 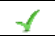 & 1 & 1 & $\checkmark$ & $\checkmark$ \\
\hline GIS & 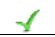 & $\mathbf{x}$ & $x$ & $\mathbf{x}$ & 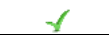 \\
\hline Maps & $d$ & $x$ & $\mathscr{d}$ & $\mathbf{x}$ & $d$ \\
\hline $\begin{array}{c}\text { Time } \\
\text { precision }\end{array}$ & $d$ & $\mathbf{x}$ & $x$ & $d$ & $\mathscr{d}$ \\
\hline $\begin{array}{l}\text { SMS and } \\
\text { GSM }\end{array}$ & $\checkmark$ & $d$ & $\mathscr{d}$ & $x$ & $\checkmark$ \\
\hline $\begin{array}{c}\text { Energy } \\
\text { Monitoring }\end{array}$ & $d$ & $x$ & $x$ & $x$ & $d$ \\
\hline $\begin{array}{c}\text { Internet } \\
\text { Requirement }\end{array}$ & $\gamma$ & $x$ & $x$ & $\mathscr{V}$ & $\gamma$ \\
\hline $\begin{array}{c}\text { Traffic } \\
\text { Management }\end{array}$ & $\gamma$ & $x$ & $x$ & $x$ & 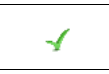 \\
\hline $\begin{array}{l}\text { Desktop and } \\
\text { Web } \\
\text { Application }\end{array}$ & $\mathscr{V}$ & $x$ & $x$ & $x$ & $\mathscr{I}$ \\
\hline $\begin{array}{l}\text { Smart Phone } \\
\text { Application }\end{array}$ & $x$ & $\mathbf{x}$ & $d$ & $\mathscr{V}$ & $\mathscr{d}$ \\
\hline
\end{tabular}

II. METHODOLOGY

The algorithm that Google adopts to find the shortest path is Dijkstra's algorithm. The idea of this algorithm is finding the shortest path from one specific node to another. This algorithm works on a weighted graph that has a start node and a goal node and the mission is finding the least cost path to the goal node. The Dijkstra's algorithm works as follows:

- In the beginning, the distance of each node will be set to $\infty$ with the exception of the first node that we will start from that is set to 0 . In addition, each node will be marked as unprocessed.

- There will be visited nodes that start with the first node and unvisited nodes that start with the rest of the nodes.

- In the current node, find out all of its neighbors that are unvisited and calculate (the distance of the current node PLUS the distance from the current node to its neighbor). If the result of this addition is less than their current temporary distance, it should be replaced with this new value.

- The node is considered to be visited and removed from the unvisited set if we are done considering all of its neighbors.

- The algorithm will stop and considered to be finished when the goal node status is changed from unvisited to visited.

- The unvisited node that has the smallest temporary distance must be marked to be the "next" to the current node and then repeat the steps starting from step 3.

Fig. 1 shows the Dijkstra's pseudo code. 


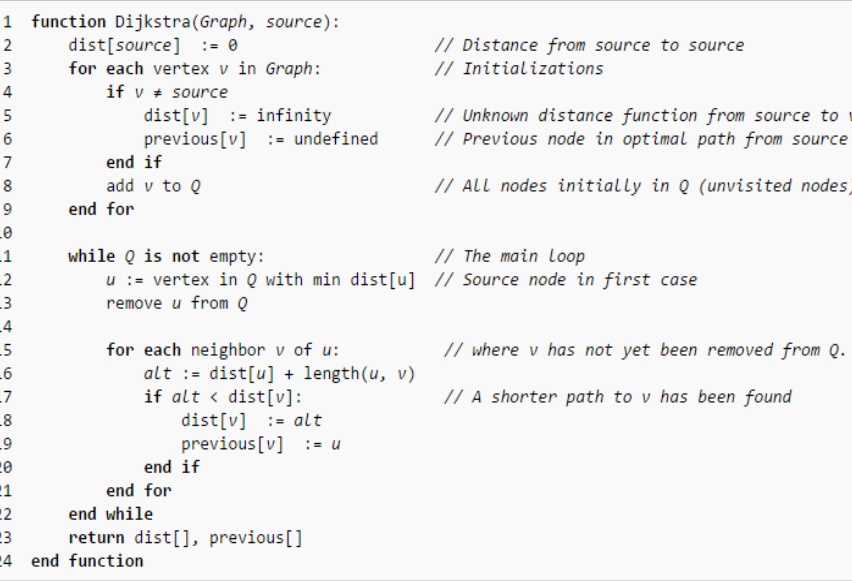

Fig. 1. Dijkstra's pseudo code

Three-tier system architecture is used for developing mobile application as shown in Fig. 2. The selection of this type of architecture is due to the following reasons. First, Three-tier architecture improves security that is needed in the application; the application layer will get the data after ensuring that a particular user has the right to access this data. Second, it facilitates in maintenance of the application. Third, it optimizes reusability.

- Data layer: It contains the local data.

- Application layer: The system uses this layer to apply the important functionality such as the operations related to logging, exception handling and validation.

- Presentation layer: It permits users to interact with our system through the user interface. It also has components that take the input from the user and validate it based on some constraints.

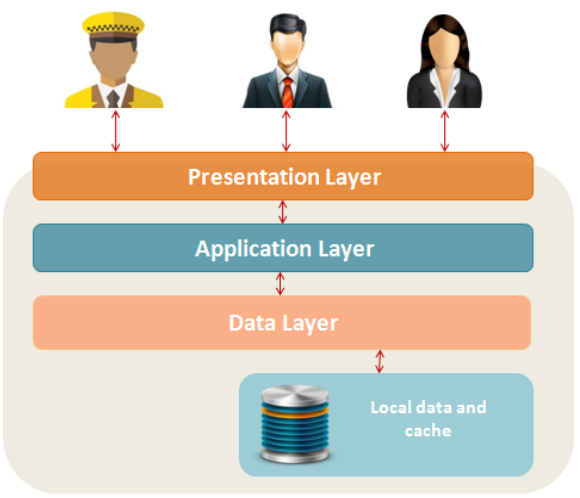

Fig. 2. System architecture

The functional requirements of the application are as shown in Fig. 3 with the help of use-case diagram.

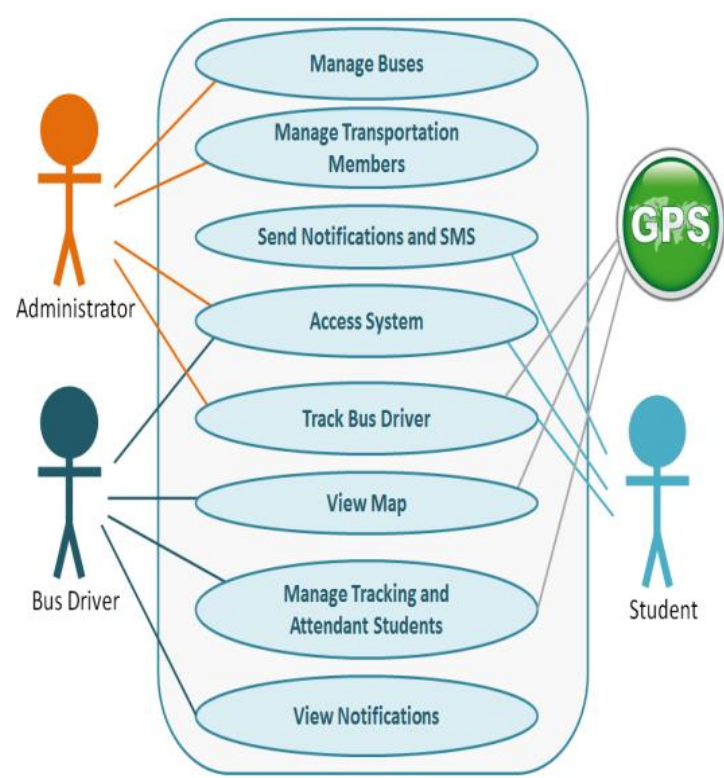

Fig. 3. Funtional requirements

\section{RESULTS}

In this project a web application is developed using PHP and mysql database to be managed by the administrator on windows platform using Apache server with Intel i7 processor, 4 GB RAM and $512 \mathrm{MB}$ hard disk. On the other end students and drivers use this information and communicate with each other using the JSON object of Android $^{8}$ operating system 2.2 or above after installing the .apk file in their mobiles with Quad Core 1.2 $\mathrm{GHz}$ processor, 1 GB RAM as minimum requirements. The developed application is tested with a sample of 30 students from Imam University, 3 buses operating on different routes handled by one administrator. Fig. 4 shows the few screen shots of web application used by the administrator. Fig. 4(a) shows the home page of the developed web application. Upon login to the system the buses related information can be viewed in buses tab as in Fig. 4(b). A new bus information can be added by filling the details related to the bus as shown in Fig. 4(c) and the confirmation message is viewed in Fig. 4(d). The transportation members tab has three options to select Fig. 4(e), upon selecting transportation administrators, the system allows to add, view, edit and delete services as shown in Fig. 4(f). Selecting bus drivers option Fig. 4(g) offers the services of add, view, edit and delete the information related to bus drivers. Similar services are offered by the system as shown in Fig. 4(j) for the student's option in Fig. 4(i). The details related to a particular student can be viewed upon clicking the view in students page Fig. 4(k). The students location on the map can be viewed as shown in Fig. 4(1) upon clicking the location on map in students page. Fig. 4(m) shows all the bus locations on the map upon selecting bus locations tab.

\footnotetext{
${ }^{8} \mathrm{http} / / / \mathrm{www} \cdot$ businessinsider.com/12-ways-android-is-still-better-than-ios-72013-9?op=1
} 


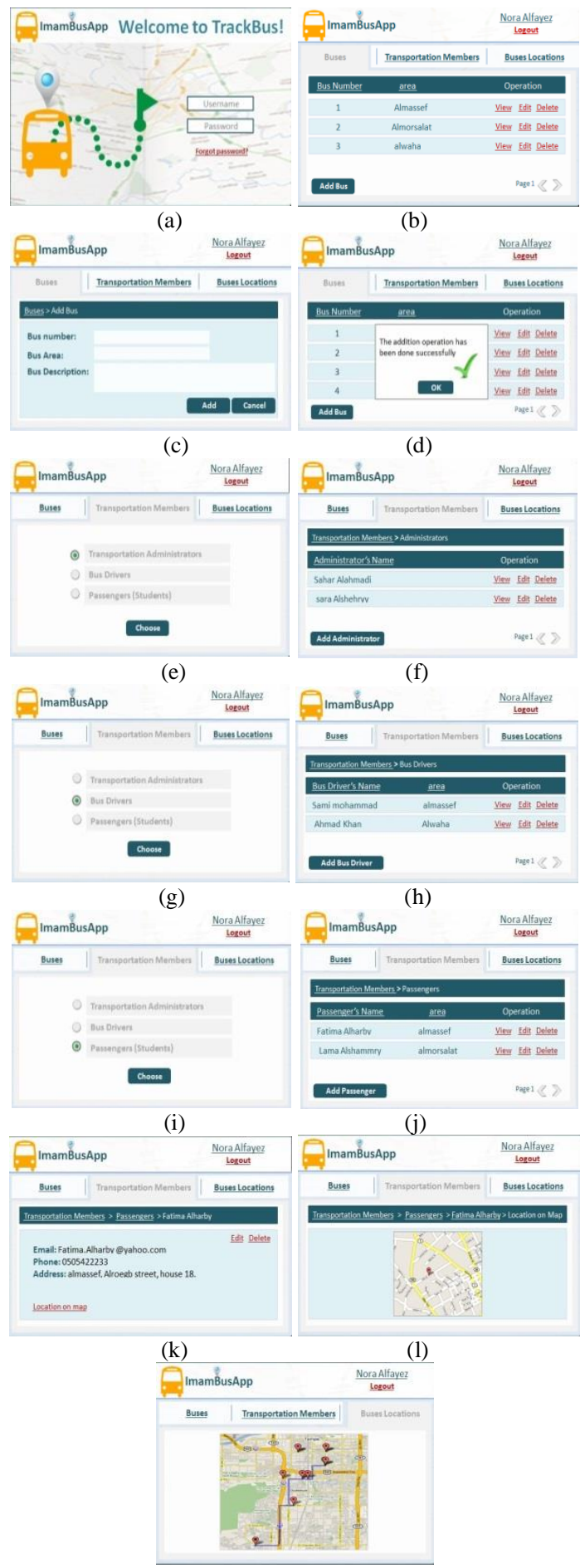

(m)

Fig. 4. Administrator functions

Fig. 5 shows the few snap shots after installing the apk file in an Android mobile used by the drivers. Fig. 5(a) shows the login screen and after successfully logged on the driver view the shortest path to the destination as shown Fig. 5(b). The driver manage the busload by adding or deleting students from Fig. 5(c) upon selecting the Manage Bus Load option from Fig. 5(a). The driver can view the location sent by the students as shown in Fig. 5(e) from selecting the view option of a particular student in Fig. 5(d) upon selecting View Students Notification service in Fig. 5(a). The driver can send the message to any selected student from selecting contact as shown in Fig. 5(f). The driver has the privilege to change his password from settings as shown in Fig. 5(g) and can view details of the application in Fig. 5(h) from About service which is at the bottom of the screen.

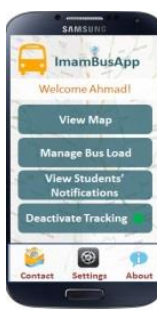

(a)

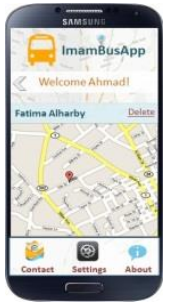

(e)

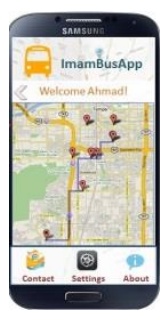

(b)

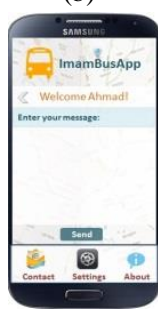

(f)

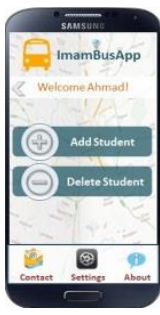

(c)

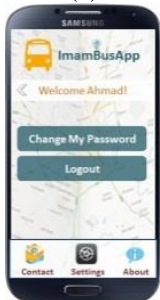

(g)

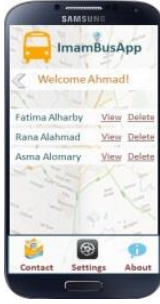

(d)

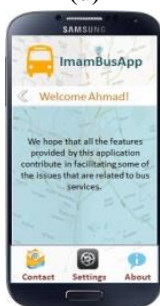

(h)
Fig. 5. Bus driver services

Fig. 6 shows few interfaces used by the students. Fig. 6(a) shows the home screen of the student upon successful login. The student can see two options, upon selecting Track My Driver in Fig. 6(a) The student can see the position, location of the driver on the map and the current bus load as shown in Fig. 6(c) and Fig. 6(d).

The students can inform the bus driver for their absence any time prior to the bus arrival to the bus-stop by selecting Absent Tomorrow service in Fig. 6(a) which can be reconfirmed by the system in Fig. 6(e) and message can be successfully delivered to the driver as shown in Fig. 6(f).

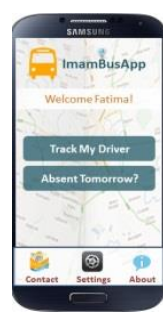

(a)

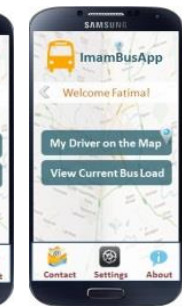

(b)

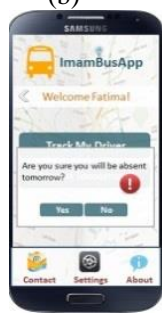

(e)

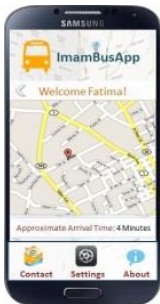

(c)

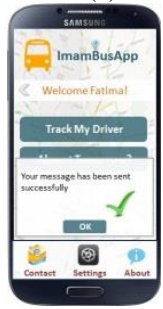

(f)

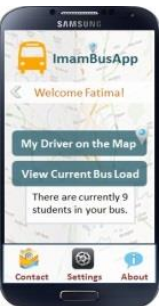

(d)
Fig. 6. Student services 


\section{CONCLUSION}

After testing the developed application a questionnaire is distributed to evaluate the students satisfaction. Very promising results with a satisfaction rate of $98.7 \%$ are achieved. This application not only helps transportation administrators of Imam University to manage transportation services that are related to buses and students efficiently and effectively but also serves vast segments of society. The developed application can be used by any transportation system and also enable parents to track their children, employers to track their employees, track the elderly people etc. It can also be utilized to register movements for the tracker himself/herself to go back and explore the places that they were in at any given time.

Enhancement to this system can be done as a part of future work by adding the following features.

- Having programmed hardware to act as a control unit that is attached to each bus. It is more convenient to track the bus vehicle itself instead of tracking the bus driver.

- Providing a more improved way of informing the student about getting into her area. Once the bus enters the area of a particular student, a notification is automatically sent or an SMS message

- Having sensors in the bus whereby, each time someone gets in, the counter of the number of the students on the bus increases. Also each time someone gets out, the counter decreases.

- Having a chat room for the students who live in the same area and who share the same bus.

- Showing the students the route of the day on a map and to give an idea of student numbers on any specific day. If many students are coming, this means the route is longer and if few students are coming, the route is shorter. Having an idea about how long the route of the day would allow the students to know when the bus may come and pick them up, thus saving them from having to wait for an unknown length of time.
Because of the fact that there are two subsystems that are collaborating with each other (the web application and the Android application) to produce the necessary information, the system's performance is not very high. This is one of the limitations of this system and finding techniques to improve the performance of the system can also be a part of the future work.

\section{REFERENCES}

[1] R. Meier, "Professional Android 4 Application Development," John Wiley \& Sons, 2012

[2] D. Huber, "Background positioning for mobile devices - android vs iphone," In Joint Conference of IEEE Computer \& Communication Societies, 2011.

[3] E. Oliver, "A survey of platforms for mobile networks research," ACM SIGMOBILE Mobile Computing and Communications Review, vol.12, pp 56-63, October 2008.

[4] O. O. Emmanuel and M. N. Moses, "GIS based public bus transport management system for Nairobi city," 1 st Esri Eastern Africa User Conference (EAUC), Nairobi, Kenya, 17-18 September, 2013.

[5] A. Kannaki, N. Vijayalashmy, V. Yamuna , G. Rupavani and G.Jeyalakshmy,"GNSS based bus monitoring and sending SMS to the passengers," International Journal of Innovative Research in Computer and Communication Engineering, vol. 2, Special Issue 1, pp. 2502 2506, March 2014.

[6] M. E. Mallia and K. Simpson, "Wireless global positioning system fleet tracking system at the university at Albany. Report No. C-11-12/ 14-27, 2014.

[7] N. Marmasse and C. Schmandt, "Save and sound : a wireless leash," In Proceedings of CHI '03 Extended Abstracts on Human Factors in Computing Systems, Ft. Lauderdale, FL, USA, April 05-10, 2003, pp. 726-727.

[8] S. Motahari, H. Zang, S. Bali, and P. Reuther, "Mobile applications tracking wireless user location," In Proceedings of IEEE Global Communications Conference (GLOBECOM), 3-7 Dec. 2012, Anaheim, CA, pp. 2006-2011.

[9] H. Lee, I. Park, and K. Hong, "Design and implementation of a mobile devices-based real-time location tracking," In The Second International Conference on Mobile Ubiquitous Computing, Systems, Services and Technologies, Sept. 29 2008-Oct. 4 2008, Valencia, pp. 178-183.

[10] M. Anisetti, V. Bellandi, E. Damiani, and S. Reale. "Advanced localization of mobile terminal," In International Symposium on Communications and Information Technologies, 17-19 Oct. 2007, Sydney, NSW, pp. 1071-1076.

[11] L. Barkuus and A. Dey, "Location based services for mobile telephony: a study of users' privacy concerns," In Proceedings of Interact 2003, Zurich, Switzerland, 2003, pp. 709-712. 